\title{
Additivity of abrupt onset effects supports nonspatial distraction, not the capture of spatial attention
}

\author{
Charles L. Folk \\ Villanova University, Villanova, Pennsylvania \\ ROGER W. REMINGTON \\ University of Queensland, Brisbane, Australia \\ AND \\ SHU-Chieh WU \\ NASA Ames Research Center, Moffett Field, California
}

\begin{abstract}
In a recent article, Schreij, Owens, and Theeuwes (2008) reported that abruptly onsetting distractors produce costs in performance even when spatial-cuing effects confirm the presence of a top-down set for color. The authors argued that these results show that abruptly onsetting new objects capture attention independent of a topdown set and, thus, provide conclusive evidence against the theory that attentional capture is contingent on topdown attentional control settings (Folk, Remington, \& Johnston, 1992). In the following article, we argue that, contrary to the conclusion drawn by Schreij et al., their own data (1) disconfirm the claim that their abrupt onsets captured spatial attention and (2) are consistent with nonspatial interference accounts of singleton-distractor effects. In support of the nonspatial account, we show that in a paradigm similar to Schreij et al.'s, distractors that do not capture attention can nonetheless influence responses to a target. We conclude that the results of Schreij et al. do not represent a challenge to contingent capture theory.
\end{abstract}

It is generally agreed that the allocation of spatial attention to objects or locations can be initiated voluntarily, in accordance with behavioral goals, or involuntarily, in response to salient stimulus events- even those events that are irrelevant to behavioral goals. The latter form of attention allocation, referred to as attentional capture, has been the subject of a debate with respect to its cognitive penetrability. Some have argued that attentional capture is a purely bottom-up process that is driven strictly by the relative salience of stimuli (e.g., Theeuwes, 1994), whereas others have argued that the capture of spatial attention is contingent on top-down attentional control settings (i.e., contingent attentional capture [CAC]; Folk, Remington, \& Johnston, 1992). Evidence for the bottom-up theoretical perspective comes primarily from the additionalsingleton paradigm (Theeuwes, 1994), whereas evidence for the top-down theoretical perspective comes primarily from the modified spatial cuing paradigm.

In a recent article, Schreij, Owens, and Theeuwes (2008) combined these two paradigms in a series of experiments that explored the influence of a top-down set on the ability of one particularly salient stimulus property - abrupt onset - to capture attention. They found that despite the presence of cuing effects confirming the presence of a top-down set for color, the appearance of an irrelevant, abruptly onsetting object simultaneous with the target produced a significant cost in performance. The authors argued that these results provide conclusive evidence that abruptly onsetting new objects capture attention independent of the top-down set and, therefore, directly challenge $\mathrm{CAC}$ theory. In the following article, we argue that, contrary to the conclusion drawn by Schreij et al., their own data (1) disconfirm the claim that their abrupt onsets captured spatial attention and (2) are consistent with nonspatial interference accounts of additional-singleton effects. As such, their results do not represent a challenge to CAC theory.

\section{The Schreij et al. (2008) Study}

The debate regarding the cognitive penetrability of attentional capture has been driven by conflicting results from two different paradigms. In the additional-singleton paradigm, irrelevant distractors presented simultaneously with the target typically produce a significant cost in performance, even when the singleton is defined by a dimension or feature value different from that of the target. Such results suggest that salient stimuli capture attention independent of the top-down set. In the modified spatial

C. L. Folk, charles.folk@villanova.edu 
cuing paradigm, irrelevant distractor precues, presented at a short interval prior to the target display, produce spatialcuing effects (i.e., faster response times [RTs] when the distractor and target are at the same location than when they appear at different locations) but only when the properties that define the precue match those that define the target. Such results suggest that the capture of spatial attention is contingent on top-down attentional control settings for the target property.

The study by Schreij et al. (2008) combined these two paradigms, in an effort to explore the effect of a top-down set for color on the ability of an abrupt-onset distractor to capture attention. Participants searched for a target of a certain color (e.g., red), which was preceded by an irrelevant distractor precue of the same color. These are conditions that have previously been shown to produce the spatial-cuing effects consistent with attentional capture by the precue (e.g., Folk et al., 1992). On half of the trials, an abruptly onsetting new-object distractor in a nontarget color appeared simultaneously with the target display at an unpredictable empty display location. In four separate experiments, irrelevant precues produced significant cuing effects, suggesting that participants had indeed adopted a set for color singleton targets. Yet, even in the presence of this top-down set for color, the appearance of an abruptly onsetting new object produced a significant cost in performance, consistent with its having captured attention. The fact that this effect was apparent when the target display was gradually onset (their Experiment 2), as well as when the nontarget characters were all different colors from the target (their Experiment 4), rules out the possibility that the participants had adopted a top-down set for displaywide onsets or a set for singletons, respectively. Finally, the identity of the character inside the abrupt-onset distractor influenced RTs to the target, with significantly faster RTs when the distractor identity matched that of the target than when it did not (their Experiment 3). Such compatibility effects are consistent with the allocation of attention to the onset distractor. The authors concluded that, taken together, their experiments provide clear and convincing evidence that abruptly onsetting new objects capture attention in a way that is independent of the topdown set (for color in this case), which is inconsistent with CAC theory.

\section{Interpreting the Data}

Schreij et al.'s (2008) conclusion that abruptly onsetting new objects capture spatial attention independent of the top-down set for color rests primarily on the combined main effects of cue validity and distractor presence. That is, the cue-validity effect establishes that a top-down control set for color is in place, and the onset-distractor effect reflects attentional capture that occurs even in the presence of that set.

The problem with the authors' account is that it does not fit the data. According to their account, upon presentation of the target display, the color target and the onset distractor compete for control of spatial attention, and the onset distractor wins this competition, drawing attention to its location even though it is not part of the attentional set.
However, this account predicts an interaction between cue validity and distractor presence, and that interaction should take a very specific form. Specifically, when there is no abrupt-onset distractor present in the target display, the effects of cue validity should be readily apparent, whereas the presence of an onset distractor in the target display should effectively eliminate the effects of cue validity. To clarify the latter prediction, consider a trial on which the precue has resulted in a shift of spatial attention to the target location (i.e., a valid cue trial). If, upon presentation of the target display containing an abruptly onsetting distractor, attention is involuntarily shifted to the location of the distractor (the Schreij et al., 2008, claim), attention should always be at a nontarget location (i.e., the location of the onset distractor), regardless of where attention was originally drawn by the precue. In other words, the presence of an abrupt-onset distractor in the target display should essentially wipe out the effects of any previous shifts of attention elicited by the precue. On distractor-present trials, then, RTs should be equal for valid and invalid trials and of about the same absolute magnitude as those in the invalid trials in the distractor-absent condition.

The data, however, tell a very different story. In all four experiments, there was absolutely no hint of an interaction between cue validity and distractor presence. Schreij et al. (2008) acknowledged this additivity in their General Discussion section but failed to recognize the problem it presents for their conclusions:

The distracting effect of the onset is relatively small (about $8-10 \mathrm{msec}$ ) and appears to be additive with cuing effects. This pattern of results implies that the distracting effect of the onset rides on top of the contingent-capture effect of the color cue, suggesting that independent of whether attention is allocated to a valid or an invalid cue, the onset captures spatial attention for a very brief time before a response to the target can be emitted. (p. 216)

The notion that the onset distractor effect "rides on top" of the cuing effect (i.e., that it is additive with cue validity) suggests that the onset affects a stage other than the stage responsible for shifts of spatial attention.

One way of salvaging the authors' interpretation would be to assume that spatial attention was indeed captured by the onset distractor but then returned to the location of the precue prior to shifting to the target. Indeed, in oculomotor capture studies, the eyes have been observed to make brief saccades to an onset stimulus, after first fixating on a color singleton, followed by a return to the color singleton (e.g., Theeuwes, Kramer, Hahn, Irwin, \& Zelinsky, 1999). However, there are several reasons why such a rubber-band effect is highly unlikely in the Schreij et al. (2008) study. First, in oculomotor studies, observers are instructed to voluntarily move their eyes to the color singleton. Thus, in contrast to the Schreij et al. experiments, observers must return to the color singleton after being captured by the onset in order to carry out the task. Given that the color precues in the Schreij et al. study were completely uninformative with respect to the target location, there would be no incentive to voluntarily return attention 
to the cued location. Second, if, as argued by the authors, attention is driven in a purely bottom-up manner by the relative salience of items in the display, then after attention is drawn to the irrelevant onset, it should then be allocated to the target location, because the target's singleton status guarantees it to be the location with the next highest salience (note that the color precue, which originally captured attention, is no longer present in the display). Thus, according to the authors' own theoretical perspective, there is no reason that spatial attention should return to a cued location that is among the least salient locations in the display. Finally, given the extensive literature on inhibition of return (IOR), as the time between precue and target processing increases, a location to which attention has been involuntarily drawn (i.e., the cued location) is, if anything, less likely to be attended again (e.g., Theeuwes \& Godijn, 2002). Granted, the effective stimulus onset asynchrony in the Schreij et al. experiments is probably too short for IOR effects: However, given that the standard functional raison d'être of IOR is to prevent reattending to stimuli, there is certainly no a priori reason to expect that attention should be more likely to return to a nonsalient location at which attention had previously been captured rather than to the next most salient location in the display (i.e., the target). Together, these considerations suggest that the observed additivity does not stem from a return of spatial attention to the cued location but, rather, reflects a form of interference that does not arise from the allocation of spatial attention to the onset distractor.

\section{Nonspatial Distraction}

If the cost in performance produced by abrupt-onset distractors does not reflect shifts of spatial attention, then what could it represent? Previous research (e.g., Folk \& Remington, 1998) has suggested that salient irrelevant singletons can have two dissociable effects on performance. If the singleton matches a current attentionalcontrol setting, it can produce an involuntary shift of spatial attention. If it does not match attentional-control settings, it does not elicit a shift of attention but can nonetheless produce a nonspatial cost analogous to a filtering cost (Kahneman, Treisman, \& Burkell, 1983). This cost is assumed to reflect a delay in the allocation of spatial attention associated with filtering salient stimuli (such as an irrelevant singleton) out of the competition for attentional resources with the target.

In the present case, we propose that the costs associated with the presence of an abrupt-onset distractor reflect filtering costs rather than shifts of spatial attention. When the abrupt-onset distractor is present, it does attempt to control attention, and therefore requires a filtering operation that is not required in the absence of a distractor, causing a delay in the allocation of attention to the target location. This interpretation is perfectly consistent with the obtained additivity of the distractor effects with cue validity. Note also that it is consistent with the Schreij et al. (2008) claim that the distractor effect "rides on top" of the cuing effect; that is, its effect is not on the actual allocation of spatial attention but on the process that determines to which object attention should be allocated.
Schreij et al. (2008) acknowledged that their distractor effects may reflect filtering costs but argued that the compatibility effects obtained in their Experiment 3 rule out this possibility. That is, the fact that the identity of the abrupt-onset distractor influenced RTs to the target was taken as evidence that spatial attention had indeed shifted to the location of the onset distractor. However, compatibility effects need not always reflect a shift of spatial attention. For example, Lavie (1995) showed that under low-load conditions (e.g., when there are relatively few stimuli in the display), unattended distractor stimuli can produce compatibility effects even when attention is focused at the target location. This effect has been attributed to the parallel allocation of attention to distractor stimuli when the resources available exceed the resource requirements of the task. Moreover, Gibson and Bryant (2008) recently showed that the compatibility effects associated with irrelevant distractors in an attentional-capture task vary as a function of the number of display items (i.e., perceptual load). Most important, Lavie and Cox (1997) found that load is associated not with the total number of stimuli on the display but with the number of salient stimuli in the display. Specifically, they found that with display size held constant, compatibility effects were obtained only when the target item "popped out" from the background items (but see Johnson, McGrath, \& McNeil, 2002, for an exception to this pattern). This finding suggests that effective set size or load is associated with the number of pop-out items in the display. In Schreij et al.'s Experiment 3, the critical displays consisted of five items, including the singleton target and the abrupt-onset distractor. Considering Lavie and Cox, the salient color singleton target and the salient onset distractor would result in an effective set size of two. Thus, under such low-load conditions, even though attention is focused at the cued location, there is enough attentional capacity left over to allow parallel processing of both target and distractor identity, leading to compatibility effects. ${ }^{1}$

Schreij et al. (2008) acknowledged the possibility of parallel processing of distractor identity in their General Discussion section but argued that "even though this is a possibility, such a paradigm would still imply that at some point, either in serial or in parallel, attentional resources were allocated to the location of the abrupt onset" (p. 217). We agree, but in our view, parallel allocation of attentional resources (whether voluntary or involuntary) does not constitute a shift of spatial attention in the sense that attentional resources are moved from one location to another. Thus, we argue that if the compatibility effects are due to parallel processing, they do not reflect the capture of spatial attention, in the sense in which the term is typically used.

\section{The Present Experiment: Evidence for Parallel Processing}

In the previous section, we argued that the compatibility effects associated with onset distractors in the Schreij et al. (2008) study most likely reflect parallel processing arising from functionally low-load conditions rather than from the capture of spatial attention. It is assumed in 
this argument that distractors that do not capture spatial attention can nonetheless produce compatibility effects when the effective display size is two. To provide independent evidence for this assumption in the context of an attentional-capture paradigm, we conducted an experiment to see whether, in a low-load (i.e., two-item) display, compatibility effects owing to the identity of a character would be observed even when conditions were such that attention was clearly directed elsewhere.

\section{METHOD}

Participants were presented with displays consisting of just two possible target locations (placeholder boxes $4.1^{\circ}$ of visual angle to the left or right of fixation). In the target display, the target character was red and the nontarget distractor was white. The target display was preceded by an uninformative red spatial precue. On half of the trials, the identity of the nontarget character in the target display was compatible with the target, and on half of the trials, it was incompatible with the target. We expected the red precue to produce attentional capture, as was found in Schreij et al. (2008). The key issue was whether the nontarget character would produce compatibility effects. Note that we could be certain that the nontarget character would not capture attention because it neither carried the finding property of the target (i.e., the color red) nor appeared as an abruptly onsetting new object. Thus, if compatibility effects were to be obtained, it would provide strong evidence to support our assumption that under low-load conditions, the identity of stimuli that do not capture attention can nonetheless affect performance.

The participants were 24 undergraduate volunteers from Villanova University. The experiment consisted of four blocks of 64 trials. Within each block, each target ("X" or "=") appeared equally often in each of the two possible locations. The precue also appeared equally often at each location, but its location was uncorrelated with target location (i.e., $50 \%$ valid cues, $50 \%$ invalid cues). For each cue-validity condition, the identity of the character appearing in the nontarget location was the same as that of the target (i.e., compatible) on half of the trials and was opposite to the identity of the target (i.e., incompatible) on the other half. In all other respects, the stimuli and procedure were identical to those used by Folk and Remington (1998).

\section{RESULTS}

Mean correct RTs as a function of cue validity and distractor compatibility are shown in Figure 1. A withinsubjects ANOVA with validity and distractor compatibility as factors yielded a significant 26 -msec effect for the for$\operatorname{mer}[F(1,23)=72.88, p<.001]$ and a 33-msec effect for the latter $[F(1,23)=66.41, p<.001]$. The trend toward overadditivity of compatibility with cue validity did not reach significance. Planned comparisons of the effect of compatibility at each level of cue validity yielded highly significant compatibility effects for both valid and invalid trials $[F(1,23)=13.67, p<.01$, and $F(1,23)=51.50$, $p<.001$, respectively]. To be certain that the obtained compatibility effects do not reflect scanning strategies associated with left-to-right reading, we reran the analysis with target position (left vs. right) as a factor. For rightside targets, the compatibility effect was $32 \mathrm{msec}$, and for left-side targets, the effect was $33 \mathrm{msec}$. The statistics confirmed that the interaction between target position and compatibility was not significant $[F(1,23)=0.04, p>$

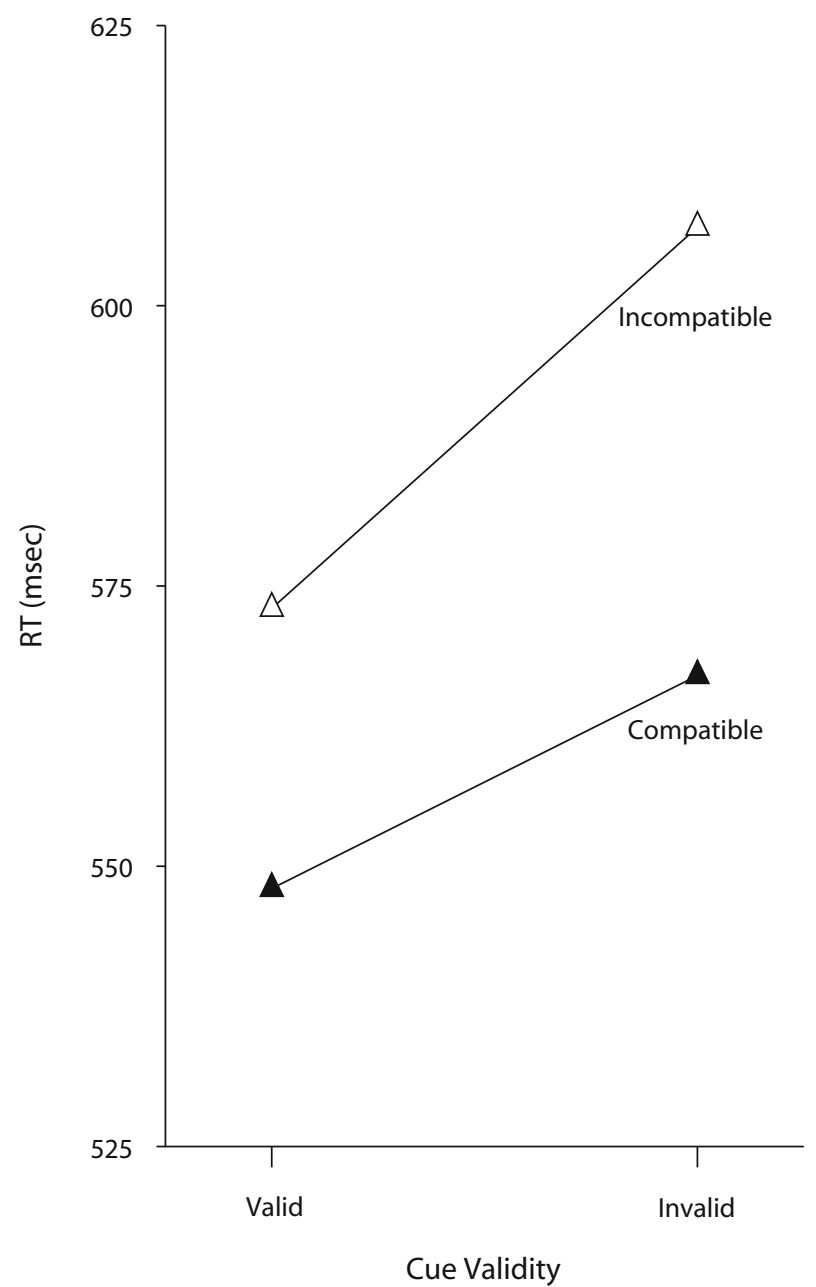

Figure 1. Mean response time (RT) as a function of cue validity and distractor compatibility.

.85], and compatibility did not enter into any interactions with cue validity. The overall error rate was $2.9 \%$, and an ANOVA with cue validity and distractor compatibility as factors yielded no significant effects.

The results of this experiment are clear. Significant main effects of both cue validity and compatibility were obtained. More important, the compatibility effect was significant for both validly (a $25-\mathrm{msec}$ effect) and invalidly (a 41-msec effect) cued trials. A compatibility effect for invalid trials is not surprising, given that the nontarget appeared at the cued location (i.e., the location to which spatial attention was drawn). What is critical is the fact that the compatibility effect was significant even on validly cued trials. In other words, even when attention was not drawn to the location of the nontarget, its identity nonetheless produced a significant compatibility effect similar in magnitude to that reported by Schreij et al. (2008).

By the logic employed by Schreij et al. (2008), the presence of this compatibility effect would mean that attention was captured by the nontarget character. However, by their logic regarding the conditions under which capture occurs, 
the nontarget character cannot capture spatial attention, because it does not carry the target color, nor is it an abruptly onsetting singleton. Thus, these results provide an existence proof that in displays consisting of two items, compatibility effects can be produced by a nontarget item that has not captured spatial attention. Given the possibility that the displays used by Schreij et al. were encoded in terms of two salient objects (i.e., the target and the abruptly onsetting new object), the present results show that the compatibility effects that they obtained could have been caused by parallel processing of low-load displays rather than by a shift of spatial attention to the distractor. Indeed, given the additivity of cue-validity and onset-distractor effects found in all four of their experiments, this seems like the most likely source of the obtained compatibility effects.

Note that although validity and compatibility effects in the present experiment were both assumed to reflect the allocation of spatial attention, we also assumed that they are generated by dissociable mechanisms similar to what Lavie, Hirst, de Fockert, and Viding (2004) referred to as active and passive control mechanisms. That is, cuing effects are assumed to reflect the capture of attentional focus, regardless of load, by stimuli carrying targetrelevant properties that are consistent with the top-down attentional set, whereas compatibility effects are assumed to reflect the nonselective (i.e., parallel) allocation of extra resources under low-load conditions, even when attention has already been focused. We suggest that the latter process is what produced the distractor-compatibility effects in the Schreij et al. (2008) experiments.

Note also that we are not arguing against the use of compatibility effects to index shifts of spatial attention. We are simply suggesting that because compatibility effects can be produced even when conditions are such that we know attention has not been allocated to the distractor location, the presence of compatibility effects is not unambiguous evidence of the focus of attention having shifted to a distractor. As pointed out by Schreij et al. (2008), we have previously used the lack of compatibility effects in the spatial-cuing paradigm to argue that abrupt-onset precues do not capture spatial attention when participants are set to respond to color targets (Folk \& Remington, 1999). The power of that conclusion, however, rested not only on the lack of compatibility effects (and the lack of cue-validity effects) when color targets were preceded by onset cues, but on the fact that significant compatibility effects (as well as cue validity effects) were found when color targets were preceded by color cues. In other words, it was the convergence of the spatial-cuing and compatibility results under different cue-target conditions that allowed us to make strong claims regarding the presence/ absence of shifts of spatial attention. If we apply this logic to the Schreij et al. experiments, in order to convincingly argue that their compatibility effects index shifts of spatial attention, one would have to show that distractors that fail to capture spatial attention fail to produce compatibility effects. However, our spatial-cuing experiment described above clearly shows that nonsalient distractors (i.e., distractors that do not capture spatial attention) can, in fact, produce such effects.

\section{CONCLUSIONS}

The experiments of Schreij et al. (2008) were an attempt to conclusively show that irrelevant, abruptly onsetting new objects can capture attention under circumstances in which independent evidence confirms the presence of a top-down set for color. At first glance, the results appear to satisfy that goal and to directly challenge the theory that attentional capture is contingent on top-down control settings. In four experiments, abruptly onsetting new objects produced costs in performance even when cuing effects established that participants had adopted a top-down set for color.

We have shown, however, that the reliable additivity of cue validity and onset presence in the Schreij et al. (2008) experiments actually provides strong evidence against their conclusion that spatial attention was captured by abruptly onsetting new objects. Accounting for this additivity in the context of the attentional capture account would require the novel assumption that attention, once captured, returns to the location of an irrelevant, inconspicuous item despite the presence of a salient singleton, a possibility that seems inconsistent with the authors' own salience-based model of attentional capture. We have provided an alternative interpretation of the Schreij et al. results that can account for the additivity as well as the obtained compatibility effects, and that is entirely consistent with the extant literature, requiring no novel assumptions. In particular, we have argued that the distracting effect of the onsets most likely represents nonspatial filtering costs similar to those found in Folk and Remington (1998) and that the compatibility effects reflect the parallel allocation of extra processing resources in the context of functionally low-load displays, similar to the effect reported by Lavie and Cox (1997). In support of the latter point, we have provided new empirical evidence that under lowload conditions, nontargets that do not capture attention can nonetheless produce compatibility effects, suggesting that the compatibility effects reported by Schreij et al. are nondiagnostic with respect to indexing shifts of spatial attention. In conclusion, contrary to the authors' claims, the results of Schreij et al. do not in any way challenge the notion that attentional capture by abruptly onsetting new objects is contingent on a top-down set.

\section{AUTHOR NOTE}

Correspondence concerning this article should be addressed to C. L. Folk, Department of Psychology, Villanova University, Villanova, PA 19085 (e-mail: charles.folk@villanova.edu).

\section{REFERENCES}

Folk, C. L., \& Remington, R. W. (1998). Selectivity in distraction by irrelevant featural singletons: Evidence for two forms of attentional capture. Journal of Experimental Psychology: Human Perception \& Performance, 24, 847-858.

Folk, C. L., \& Remington, R. W. (1999). Can new objects override attentional control settings? Perception \& Psychophysics, 61, 729-739.

Folk, C. L., Remington, R. W., \& Johnston, J. C. (1992). Involuntary covert orienting is contingent on attentional control settings. Journal of Experimental Psychology: Human Perception \& Performance, 18, 1030-1044. 
Gibson, B. S., \& BRYAnT, T. A. (2008). The identity intrusion effect: Attentional capture or perceptual load. Visual Cognition, 16, 182-199.

Johnson, D. N., McGrath, A., \& McNeil, C. (2002). Cuing interacts with perceptual load in visual search. Psychological Science, 13, 284-287.

Kahneman, D., Treisman, A., \& Burkell, J. (1983). The cost of visual filtering. Journal of Experimental Psychology: Human Perception \& Performance, 9, 510-522.

Lavie, N. (1995). Perceptual load as a necessary condition for selective attention. Journal of Experimental Psychology: Human Perception \& Performance, 21, 451-468.

Lavie, N., \& Cox, S. (1997). On the efficiency of visual selective attention: Efficient visual search leads to inefficient distractor rejection. Psychological Science, 8, 395-398.

Lavie, N., Hirst, A., de Fockert, J. W., \& Viding, E. (2004). Load theory of selective attention. Journal of Experimental Psychology: General, 133, 339-354.

Schreis, D., Owens, C., \& Theeuwes, J. (2008). Abrupt onsets capture attention independent of top-down control settings. Perception \& Psychophysics, 70, 208-218.

THEeUwES, J. (1994). Stimulus-driven capture and attentional set: Se- lective search for color and visual abrupt onsets. Journal of Experimental Psychology: Human Perception \& Performance, 20, 799-806.

Theeuwes, J., \& GodiJn, R. (2002). Irrelevant singletons capture attention: Evidence from inhibition of return. Perception \& Psychophysics, 64, 764-770.

Theeuwes, J., Kramer, A. F., Hahn, S., Irwin, D. E., \& Zelinsky, G. J. (1999). Influence of attentional capture on oculomotor control. Journal of Experimental Psychology: Human Perception \& Performance, 25, 1595-1608.

\section{NOTE}

1. Filtering costs, as originally conceptualized by Kahneman et al (1983), reflect the time it takes the system to determine that spatial attention should be allocated to the target, rather than to the distractor. In this sense, the distractor is filtered out of the competition for spatial attention. This need not imply, however, that other aspects of the distractor (e.g., its identity) are not processed.

(Manuscript received February 29, 2008; revision accepted for publication August 10, 2008.) 